IP Periodica Polytechnica Civil Engineering

\author{
62(1), pp. 250-258, 2018 \\ https://doi.org/10.3311/PPci.10420 \\ Creative Commons Attribution (i)
}

RESEARCH ARTICLE

\section{Reliability-based Support Optimization of Rockbolt Reinforcement around Tunnels in Rock Masses}

\author{
Hongbo Zhao ${ }^{1 *}$, Zhongliang $\mathrm{Ru}^{1}$, Changxing Zhu ${ }^{1}$
}

Received 16 December 2016; Revised 03 February 2017; Accepted 10 September 2017

\begin{abstract}
Traditionally, the design of tunnels is based on determinate parameter values. In practice, both the performance and safety of tunnels are affected by numerous uncertainties: for example, it is difficult for engineers to predict uncertainties in geological conditions and rock mass properties. The purpose of reliability-based optimization (RBO) is to find a balanced design that is not only economical but also reliable in the presence of uncertainty. In the past few decades, numerous reliability optimization techniques have been proposed for taking uncertainty into account in the design of engineering structures. In the present study, the first-order reliability method (FORM) was used to compute the reliability index using Excel Solver. The least squares support vector machine (LSSVM) approach was adopted to build a relationship between reliability index and design variables, and the artificial bee colony $(A B C)$ algorithm was employed for the reliability-based optimization. A proposed LSSVM/ABC-based reliability optimization method was applied to the case of a tunnel with rockbolt reinforcement. The mechanical parameters of the rock mass, in-situ stress and internal pressure were considered as the random variables. The reliability index of tunnel was analysed. The length, distance out of plane and the number of rockbolts were determined and optimized considering the uncertainty based on RBO. The proposed method improved the efficiency of RBO while maintaining high accuracy. The results showed that the proposed method not only meets the design accuracy, but also improves the efficiency of reliability-based optimization.
\end{abstract}

\footnotetext{
Keywords

reliability-based optimization, reliability analysis, first-order reliability method, artificial bee colony, least squares support vector machine

' School of Civil Engineering, Henan Polytechnic University, Jiaozuo, 454003, People's Republic of China

* Corresponding author, e-mail: bxhbzhao@hotmail.com
}

\section{Introduction}

The design and performance of tunnels are usually affected by some uncertainties that can be costly and time-consuming for tunnel construction projects. Traditional empirical and deterministic design approaches do not include uncertainty in tunnel support design [1-3], but tend to be based on trial-anderror processes that consider safety and cost [4-6]. Reliabilitybased optimization ( $\mathrm{RBO}$ ) makes provision for the uncertainty of structures by adding probabilistic constraints. This is quite straight forward if the results of the reliability analysis are accurate and precise so that no question arises as to whether a given design satisfies safety requirements. The purpose of $\mathrm{RBO}$ is to find a balanced design that is not only economical but also reliable in the presence of uncertainty [7].

Over the past few decades, numerous reliability optimization techniques have been proposed $[6,8,9]$. Younes and Alaa overviewed the various RBDO approaches using mathematical and finite element models with different levels of difficulties [10]. Marcos and Gerhart (2010) produced a detailed literature review on reliability-based optimization [11]. Although RBO has some evident advantages overdeterministic optimization design, it is often computationally inefficient. Response surface methodology has been applied in RBO in attempts to improve its efficiency $[12,13]$. Zhang et al. applied the mean first-order reliability method (MFORM) to the optimization of geotechnical systems [6]. Those methods improved the computational efficiency but decreased the accuracy of the reliability analysis, which affects the results of RBO. The selection of an optimization method is critical to RBO applications, especially for complex nonlinear optimization problems. Gen and Yun reviewed the application of soft computing methods in reliability optimization [14]. Genetic algorithms and particle swarm optimization have also been applied to RBO [7, 15]. Lee et al. proposed a methodology to convert an RBDO problem requiring very high reliability to an RBDO problem requiring relatively low reliability by appropriately increasing the input standard deviations for efficient computation in sampling-based RBDO [16]. 
In this paper we propose a LSSVM/ABC-based reliability optimization approach to the design of tunnel support, which meets the requirements both of efficiency and accuracy. The artificial bee colony (ABC) [17] was used to search the design variables in global search space. The ABC algorithm, which simulates the intelligent foraging behaviour of honey bee swarms, was originally proposed for optimizing numerical problems. It is a very simple, robust and population-based stochastic optimization algorithm. To improve efficiency, LSSVM was adopted to establish the relationship between the reliability index and the design variables. The design variables and objective function were computed by the proposed method. The results were verified by comparison with other methods, and they indicate that the proposed method provides reliability-based optimization with both high efficiency and accuracy.

\section{Background}

\subsection{Analytical solution of a circular tunnel with rockbolt reinforcement}

A radial cross-section through the rockbolt-reinforced tunnel to be designed is shown in Fig. 1: a straight tunnel of radius $a$ was to be excavated in a rock mass subject to a uniform farfield stress $\sigma_{0}$ [18]. The rock mass was assumed to be isotropic, homogeneous and elastic, with shear modulus $G$ and Poisson's ratio $\mu$. The tunnel was to be reinforced with $n_{b}$ equally spaced rockbolts of diameter $d_{b}$ and length $L_{b}$.

The plane strain displacement solution for the tunnel wall considers the cases of both grouted and anchored rockbolts is illustrated in Fig. 1. The displacements for region B when $p_{s} \leq p_{f}$ for both grouted and anchored rockbolts are given by Carranza-Torres (2009) as:

$$
u_{\mathrm{r}} \frac{2 G}{a \sigma_{0}}=u_{r}^{I N I T} \frac{2 G}{a \sigma_{0}}+\left(1-\frac{\sigma_{r}^{*}}{\sigma_{0}}\right) \frac{\rho}{\rho^{* 2}}-\left(1-\frac{p_{f}}{\sigma_{0}}\right) \rho
$$

Where $u_{r}^{I N I T}$ is the initial radial displacement before installation of the rockbolt, and $\sigma_{r}{ }^{*}$ is the radial stress at the boundary between regions A and B in Fig. 1 [18].

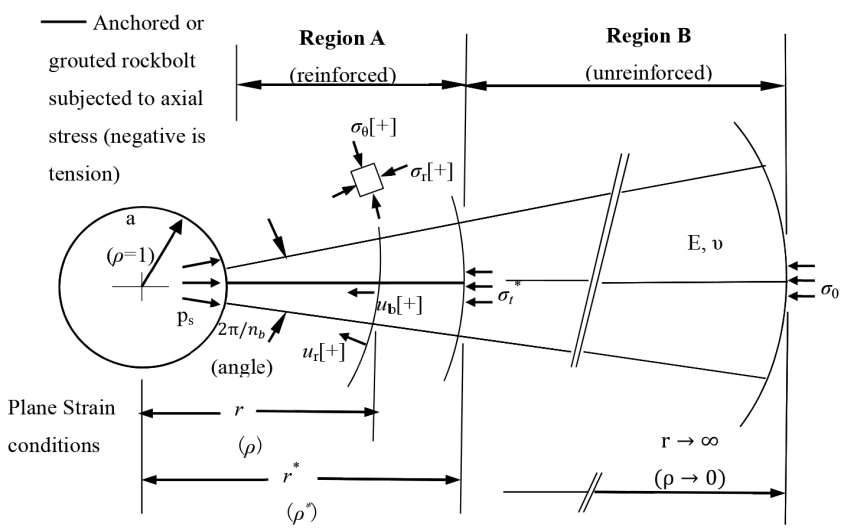

Fig. 1 Definition of variables in the axisymmetrical problem of a straight reinforced circular tunnel

In the convergence-confinement method of tunnel support design, $u_{r}^{I N I T}=0$. In other situations, $u_{r}^{I N I T}$ can be calculated from:

$$
u_{\mathrm{r}}^{\mathrm{INIT}} \frac{2 G}{a \sigma_{0}}=\left(1-\frac{p_{f}}{\sigma_{0}}\right) \rho
$$

The displacements in region A when reinforced by grouted rockbolts is given by:

$$
\begin{aligned}
u_{r} \frac{2 G}{a \sigma_{0}}= & u_{r}^{I N I T} \frac{2 G}{\sigma_{0}}+\left[1-\frac{1}{\rho} \frac{1+\mu}{\rho} \ln \left(\frac{1+\mu+\beta \rho}{1+\mu}\right)\right] \\
& \times \frac{N_{1}+N_{2}\left(\sigma_{r}^{*} / \sigma_{0}\right)}{N}-\frac{1}{\rho} \frac{1+\mu}{\rho} \frac{N_{3}+N_{4}\left(\sigma_{r}^{*} / \sigma_{0}\right)}{N}
\end{aligned}
$$

The radial stress $\sigma_{r}^{*}$ at the boundary of regions A and B for grouted rockbolts is given by:

$\frac{\sigma_{r}^{*}}{\sigma_{0}}=\frac{N_{3}(1+\mu)+\beta N \rho^{* 2}\left(p_{f} / \sigma_{0}\right)-2 \beta N_{1} \rho^{*}+2 N_{1}(1+\mu) \ln \left({ }^{1+\mu+\beta \rho^{*}} / 1+\mu\right)+N \beta\left(1-\rho^{* 2}\right)}{\beta N-N_{4}(1+\mu)+2 \beta N_{2} \rho^{*}-2 N_{2}(1+\mu) \ln \left({ }^{1+\mu+\beta \rho^{*}} / 1+\mu\right)}$

Where the coefficients $N 1, N 2, N 3, N 4, N$ for grouted rockbolts are given as follows:

$$
\begin{gathered}
N_{1}=-(1+\mu)(1+2 \mu+\beta)\left(1-\rho^{* 2}\right)+(1+\mu)(1+2 \mu)\left(1-\rho^{* 2}\right)\left(p_{f} / \sigma_{0}\right) \\
+\beta(1+\mu)\left(1-\rho^{*}\right) \rho^{*}\left(p_{f} / \sigma_{0}\right)+\alpha(1+\mu)(1+2 \mu)\left(1-\rho^{*}\right)\left(\sigma_{b}^{P S} / \sigma_{0}\right) \\
-(1+\mu)\left(1+2 \mu+\beta \rho^{*}\right)\left(\left(p_{s} / \sigma_{0}\right)\right) \\
N_{2}=(1+\mu)(1+2 \mu+\beta)
\end{gathered}
$$




$$
\begin{aligned}
N_{4}=2 \beta(1+2 \mu)-2(1+\mu)(1+2 \mu+\beta) \ln \left(\frac{1+\mu+\beta}{1+\mu}\right) & \\
N_{3}= & -2(1+\mu)(1+2 \mu)^{2}\left(1-\rho^{*}\right) \\
& -(1+\mu)^{2}(1+2 \mu+\beta)\left(1+2 \mu+\beta \rho^{*}\right) \frac{1}{\beta} \ln \left(\frac{1+\mu+\beta \rho^{*}}{1+\mu+\beta}\right)
\end{aligned}
$$

The axial stress in anchored rockbolts is given by:

$$
\sigma_{\mathrm{b}} \frac{1}{\sigma_{0}}=-\frac{2 G}{\sigma_{0}} \frac{\beta}{\alpha} \frac{\rho^{*}}{1-\rho^{*}} \frac{u_{r}(l)-u_{r}\left(\rho^{*}\right)}{a}+\frac{\sigma_{b}^{P S}}{\sigma_{0}}
$$

and in grouted rockbolts by:

$$
\sigma_{b} \frac{1}{\sigma_{0}}=-\frac{2 G}{\sigma_{0}} \frac{\beta}{\alpha} \frac{\rho^{2}}{a}\left(u_{r}^{\prime}-u_{r}^{I N I T^{\prime}}\right)+\frac{\sigma_{b}^{P S}}{\sigma_{0}}
$$

\subsection{FORM algorithms}

The reliability index is a measure of the stability of engineering systems that takes into account the inherent uncertainty of input variables. The widely used Hasofer-Lind index, $\beta$ is defined as the minimum distance from the mean value point of the random variables to the boundary of the limit state surface [19], in directional standard deviation units. It is expressed as follows:

$$
\beta=\min _{X \in F} \sqrt{(X-\mu)^{T} C^{-1}(X-\mu)}
$$

Where $X$ is a vector representing the set of random variables $x_{i} ; \mu$ is a vector of mean values; $C$ is the covariance matrix; and $F$ is the failure domain. Low and Tang (2007) proposed anefficient algorithm for FORM [20], which changes the dimensionless number $n_{i}$, so that Eq. (12) maybe rewritten as:

$$
\beta=\min _{X \in F} \sqrt{[n]^{T}[R]^{-1}[n]}
$$

Where $R$ is the correlation matrix and where $n$ is a column vector of $n_{i}$ given by:

$$
n_{i}=\frac{x_{i}-\mu_{i}^{N}}{\sigma_{i}^{N}}
$$

Where $\mu_{i}^{N}$ and $\sigma_{i}^{N}$ are the equivalent normal mean and equivalent normal standard deviation, respectively, for random variable $x_{i}$.

\subsection{Reliability analysis of a rockbolted circular tunnel}

In this study, Low and Tang's (2007) method was used to compute the reliability index using Excel Solver. The elastic modulus $\mathrm{E}$, in-situ stress $\sigma_{0}$ and $p_{f}$ are the random variables, and the other parameters are determinate variables. To investigate the reliability analysis of a circular tunnel with rockbolt reinforcement, the performance function is given by the displacement solutions of the tunnel wall and the radial stress in the rockbolt:

$$
\begin{aligned}
& G_{1}(x)=\varepsilon_{L}-u_{r}^{w} \\
& G_{2}(x)=\sigma_{r}-\sigma_{r}^{T}
\end{aligned}
$$

Where $\varepsilon_{L}$ is the limiting inward displacement of the tunnel wall; $u_{r}{ }^{w}$ is the deformation criterion; $\sigma_{r}$ is the radial stress in the rockbolt; and $\sigma_{r}{ }^{T}$ is the tensile strength of the rockbolt. The performance function becomes negative when the inward displacement of the tunnel wall $u_{r}{ }^{w} \geq \varepsilon_{L}$.

$\varepsilon_{L}$ and $\sigma_{r}$ were calculated from Eqs.(1) and (11)using Microsoft Visual Basic for Applications (VBA). Fig. 2 shows a typical Excel spreadsheet for computation of the reliability index for the tunnel when considering two failure modes: the deformation exceedance criterion, and rockbolt failure.

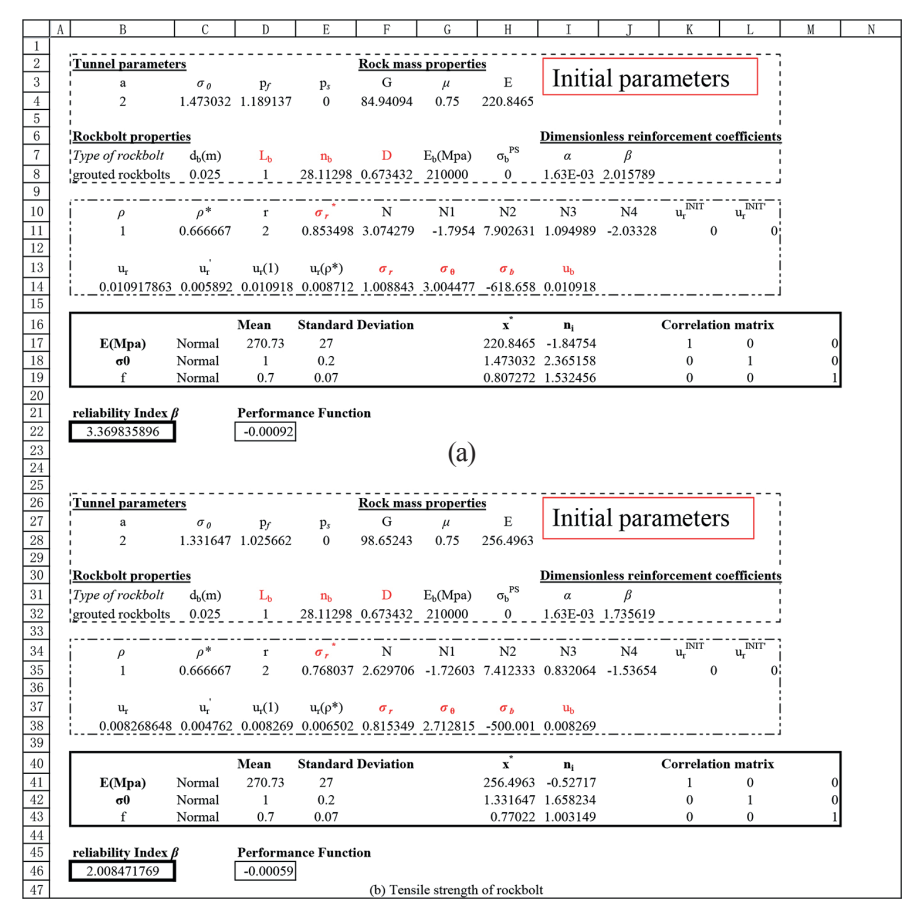

(b)

Fig. 2 Reliability index using Excel Solver, based on: (a) surrounding deformation; (b) tensile strength of rockbolt

\subsection{Least squares support vector machine (LSSVM)}

LSSVM is an alternative formulation of the Suykens and Vandewalle SVM regression method [21] of establishing the relationship between reliability index and design variables. Consider a given training set of $N$ data points $\left\{x_{k}, y_{k}\right\}$ $(k=1,2, \ldots, N)$ with input data $x_{k} \in R^{N}$ and output $y_{k} \in R$ where $R^{N}$ is the $N$-dimensional vector space and $R$ is the one-dimensional vector space. The LSSVM model is given by:

$$
y(x)=\sum_{k=1}^{N} \alpha_{k} K\left(x, x_{k}\right)+b
$$

Where $k\left(x, x_{k}\right)$ is the kernel function; $\alpha$ is the Lagrangian coefficient; and $b$ is the scalar threshold. The values of $\alpha$ and $b$ are obtained from:

$$
\left[\begin{array}{cc}
0 & 1^{T} \\
1 & \Omega+\gamma^{-1} I
\end{array}\right]\left[\begin{array}{l}
b \\
\alpha
\end{array}\right]=\left[\begin{array}{l}
0 \\
y
\end{array}\right]
$$

where $y=\left[y^{1}, \ldots, y_{N}\right] ; I=[1, \ldots, 1], \Omega=k\left(x_{k}, x_{l}\right) ; k, l=1, \ldots, N$. Choosing $\gamma>w$ ensures that the matrix:

$$
\Phi=\left[\begin{array}{cc}
0 & 1^{T} \\
1 & \Omega+\gamma^{-1} I
\end{array}\right]
$$


is invertible. The analytical solution of $\alpha$ and $b$ is then given by:

$$
\left[\begin{array}{l}
b \\
\alpha
\end{array}\right]=\Phi^{-1}\left[\begin{array}{l}
0 \\
y
\end{array}\right]
$$

The three general kernel functions are: the polynomial kernel $K(X, Y)=((X \cdot Y)+1)^{d}, d=1,2, \ldots, n$

the radial kernel $K(X, Y)=\exp \left\{-\frac{|X-Y|^{2}}{\sigma^{2}}\right\}$

and the sigmoidal kernel $K(X, Y)=\tanh (\phi(X \cdot Y)+\theta)$.

\subsection{Artificial bee colony ( $A B C)$ algorithm}

In the $\mathrm{ABC}$ algorithm(Karaboga,2005), which is a swarmbased, meta-heuristic algorithm, the colony of imaginary bees consists of three categories of bee: employed bees, onlookers and scouts. The position of a food source represents a possible solution for the problem under consideration, and the amount of nectar in the food source represents the quality of the solution based on its 'fitness' value $[22,23]$. In the minimization problem, the fitness can be computed by the objective function.

Half of the colony consists of employed bees and the other half consists of onlookers. The numbers of employed and onlooker bees is equal to the number of solutions in the population. In the first step, the algorithm generates a randomly distributed initial population of solutions and the fitness of each solution is calculated from:

$$
x(i, j)=x_{\min }^{j}+\operatorname{rand}(0,1)\left(x_{\max }^{j}-x_{\min }^{j}\right)
$$

where $x(i, j)$ is the candidate solution of problem; $i=1,2, \ldots, S N$ in which $S N$ denotes the population size; $j=1,2, \ldots, D$ in which $D$ is the dimension number of each solution; $\operatorname{rand}(0,1)$ is a random number between 0 and 1 ; and $x^{j}{ }_{\min }$ and $x^{j}{ }_{\text {max }}$ are the lower and upper bounds of each solution.

After initialization, the search carried out by the bees maybe summarized as follows:

- Employed bees locate a food source within the neighbourhood of the food source in their memory.

- Employed bees share their information with onlooker bees in the hive, and then the onlookers select one of the food sources.

- Onlookers select a food source within the neighbourhood of the food sources chosen by them.

- When an employed bee abandons the food source, it becomes a scout and starts a random search for a new food source.

To produce a candidate food location from the old one in memory, the algorithm uses the following expression:

$$
v(i, j)=x(i, j)+\varphi_{i j}(x(i, j)-x(k, j))
$$

where $\mathrm{k} \in\{1,2, \ldots, S N\}$ and $\mathrm{j} \in\{1,2, \ldots, D\}$ are randomly chosen indexes. Although $k$ is determined at random, it must be different from $i$. $\phi_{i j}$ is a random number between -1 and +1 . It controls the production of neighbouring food sources around $x(i, j)$, and represents the comparison between two food positions visible to a bee. It is seenfrom Eq. (22) that, as the difference between the parameters of the $x(i, j)$ and $x(k, j)$ decreases, the perturbation on the position $x(i, j)$ therefore also decreases. Thus, as the search approaches the optimum solution within the search space, the step length is adaptively reduced.

An onlooker bee chooses a food source based on the probability $p_{i}$ that the food will be in a selected position. This is calculated by:

$$
p_{i}=\frac{\text { fitness }_{i}}{\sum_{n=1}^{S N} \text { fitness }_{n}}
$$

Where fitness $_{i}$ is the fitness of the solution.

In $\mathrm{ABC}$, a food source position that cannot be improved further through a predetermined number of cycles is assumed to be abandoned. Therefore, the value of the predetermined number of cycles, termed the "limit for abandonment", is an important control parameter in the algorithm. Assuming that the abandoned source is $x(i, j)$, the scout discovers a new food source and replaces it with an updated value of $x(i, j)$. This operation is defined by Eq.(21) above.

Once each candidate source position $v(i, j)$ is locatedas in Eq. (22), it is evaluated by the bee and its performance is compared with the previous $v(i, j)$. If the new food source contains nectar that is equal to or better than the previous source, it replaces the earlier one in the memory.

The three control parameters in the $\mathrm{ABC}$ algorithm are: the number of food sources, equivalent to the number of employed or onlooker bees $(S N)$; the value of the limit of abandonment; and the maximum cycle number $(M C N)$.The $\mathrm{ABC}$ flowchart is shown in Fig.3.Briefly, the $\mathrm{ABC}$ algorithm procedure is as follows:

Step 1: Determine the values of the control parameters $S N$, $M C N$ and abandonment limit.

Step 2: Generate the initial population $x(i, j)$ from (Eq.(21)) and evaluate the fitness of each solution.

Step 3: For each employed bee, generate new solution $v(i, j)$ from Eq.(22) and evaluate its fitness.

Step 4: Calculate the probability values $p_{i}$ for the solution $x(i$, j) by Eq.(23).

Step 5: For each onlooker bee, select a solution $x(i, j)$ and, according to $p_{i}$, produce a new solution $v(i, j)$ and calculate its fitness.

Step 6: If a solution is abandoned by the scout, a new random solution is generated by Eq.(22).

Step 7: Memorize the best solution.

Step 8: Repeat Steps 3 to 7 until $M C N$ is reached. 


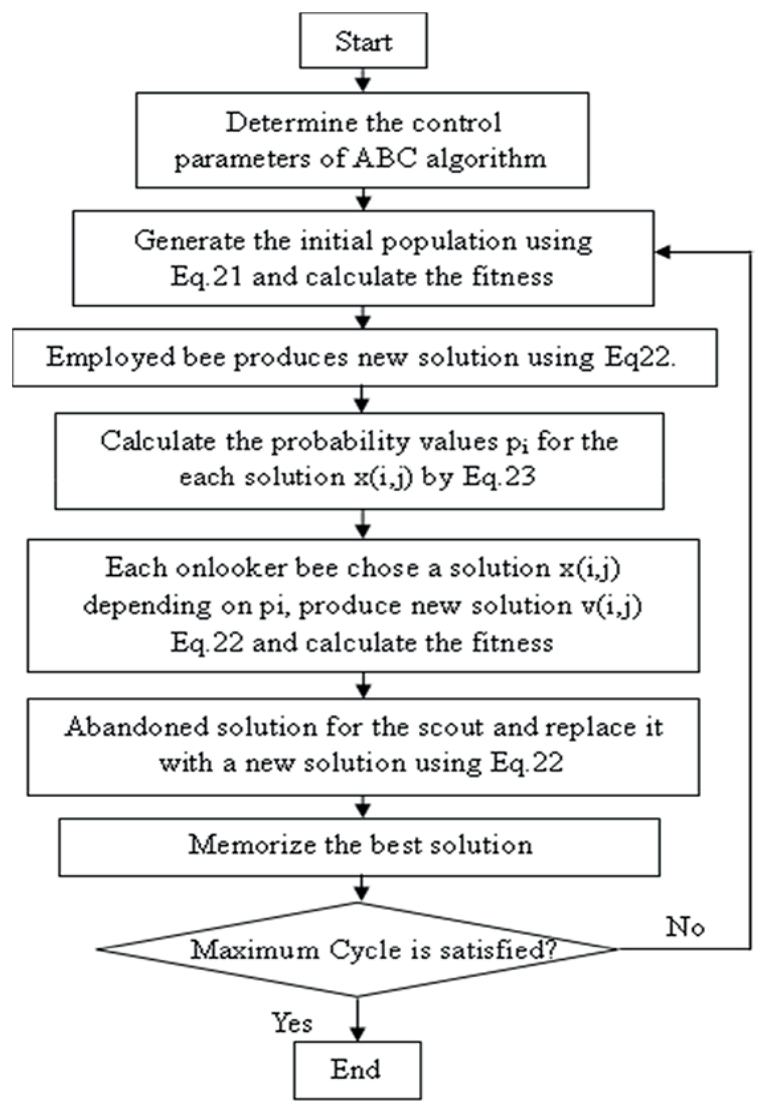

Fig. 3 Artificial bee colony (ABC) algorithm flowchart

\section{Reliability-based optimization using LSSVM/ABC 3.1 Reliability-based design optimization}

Let $d$ denote the design variables in the tunnel engineering, and let $C(d)$ denote the cost function of the design. Suppose the design requirement is that the reliability index for the $i$ th failure mode is not less than a target reliability index, $\beta_{i}^{T}$. Then, let $d_{i}$ be the $i$ th element of $d$, and $d_{i}^{L}$ and $d_{i}^{U}$ be the lower and upper permissible values of $d_{i}$. The purpose of reliability-based optimization is to find a set of design variables that minimizes the cost function, $C(d)$, without violating any design requirements. Mathematically, this problem can be expressed as:

$$
\begin{array}{llc}
\min C(d) & & \\
\text { subject to } & \beta_{i}(d)>\beta_{i}^{T} & i=1,2, \ldots, n_{m} \\
& d_{i}^{L}<d_{i}<d_{i}^{u} & i=1,2, \ldots, n_{d}
\end{array}
$$

where $C(d)$ is the objective function, such as $\operatorname{cost} ; \beta_{i}(d)$ and $\beta_{i}^{T}$ are the reliability index and reliability constraint of the $i$ th failure model; $d_{i}$ represents the design variables, for which $d_{i}^{L}$ and $d_{i}^{U}$ are respectively the lower and upper bounds of the $i$ th design variables; and $n_{m}, n_{d}$ are the number of reliability constraints and design variables. RBO differs from deterministic optimization in that it involves reliability constraints. In this study, FORM was used to compute the reliability index based on Low and Tang's (2007) method, i.e. Eq. (13).

\subsection{LSSVM-based relationship between reliability index and design variables}

The algorithm of Low and Tang (2007) computed the reliability index using Excel Solver in this work. Then, the ABC algorithm was used to search the design variables based on the objective function. LSSVM/ABC-based RBO is composed of an inner and outer loop. The inner loop mainly computes the reliability based on LSSVM. The outer loop searches the design variables using the $\mathrm{ABC}$ algorithm. The flowchart of the proposed method is shown in Fig. 4.

The nonlinear relationship between reliability index and design variables is described by the LSSVM model, stated as:

$$
y(X)=\operatorname{LSSVM}(X)
$$

Where $y(X)$ is the reliability index, and $X$ represents the design variables.

The LSSVM model may also be expressed by:

$$
y(x)=\sum_{k=1}^{N} \alpha_{k} K\left(x, x_{k}\right)+b
$$

LSSVM possesses other powerful regression capabilities. In order to obtain $\operatorname{LSSVM}(X)$, a training process based on a known dataset is needed. Training samples maybe acquired by using reliability analysis for a given set of tentative design variables to obtain the corresponding reliability index. In this study, FORM was used in Excel Solver to compute the reliability based on different performance functions.

\subsection{Fitness function}

In the $\mathrm{ABC}$ algorithm, the fitness function needed to search for the optimal value in a large search-space RBO problem can be converted into the following non-constrained optimization form by penalty method:

$$
\text { Minimize } \quad C(d)+M \cdot \sum_{i=1}^{n_{m}} f_{i}(d)
$$

Where $f_{i}(d)$ is the penalty function and $M$ is the penalty coefficient. The choice of the penalty coefficient $M$ is crucial for improving the convergence and accuracy of Eq. (27).In this study, $f_{i}(d)$ was defined as:

$$
f_{i}(d)= \begin{cases}0 & \beta_{i}^{T} \geq \beta_{i}(d) \\ \left|\beta_{i}^{T}-\beta_{i}(d)\right| & \beta_{i}^{T}<\beta_{i}(d)\end{cases}
$$

The fitness function of LSSVM/ABC-based RBO is defined as follows:

$$
\text { fitness }=C(d)+M \cdot \sum_{i=1}^{n_{m}} f_{i}(d)
$$

\subsection{Procedure of LSSVM/ABC-based reliability optimization}

In this study, the reliability index was computed by combining the FORM method with Excel Solver. The Latin hypercube sampling(LHS) statistical sampling method [24] was used to build the set of input pairs of training samples, and the nonlinear mapping relationship between the reliability index 
and design variables was built by LSSVM. ABC searched the optimal design variables based on the fitness function. A single loop is used in the LSSVM/ABC-based reliability optimization method, which improves its efficiency and reduces the time taken to complete the optimization. The flowchart is shown in Fig. 4. The stepwise procedure is as follows:

Step 1: Set the range of design variables for the problem.

Step 2: Produce the input pairs of training samples using LHS.

Step 3: Set the random variables and statistical parameters.

Step 4: Compute the reliability index of each input pair using the FORM algorithm in Excel Solver.

Step 5: Build the training samples using the input pairs (design variables) and output (reliability index).

Step 6: Build the relationship between design variables and reliability index using the LSSVM algorithm.

Step 7: Determine the parameters of ABC.

Step 8: Produce the initial bee colony population.

Step 9: Compute the fitness of individual bees based on the LSSVM model.

Step 10: Check number of cycles. END if maximum cycle has been reached; if not, GOTO Step 11.

Step 11: Update the population of bee colony using ABC algorithm, GOTO Step 9.

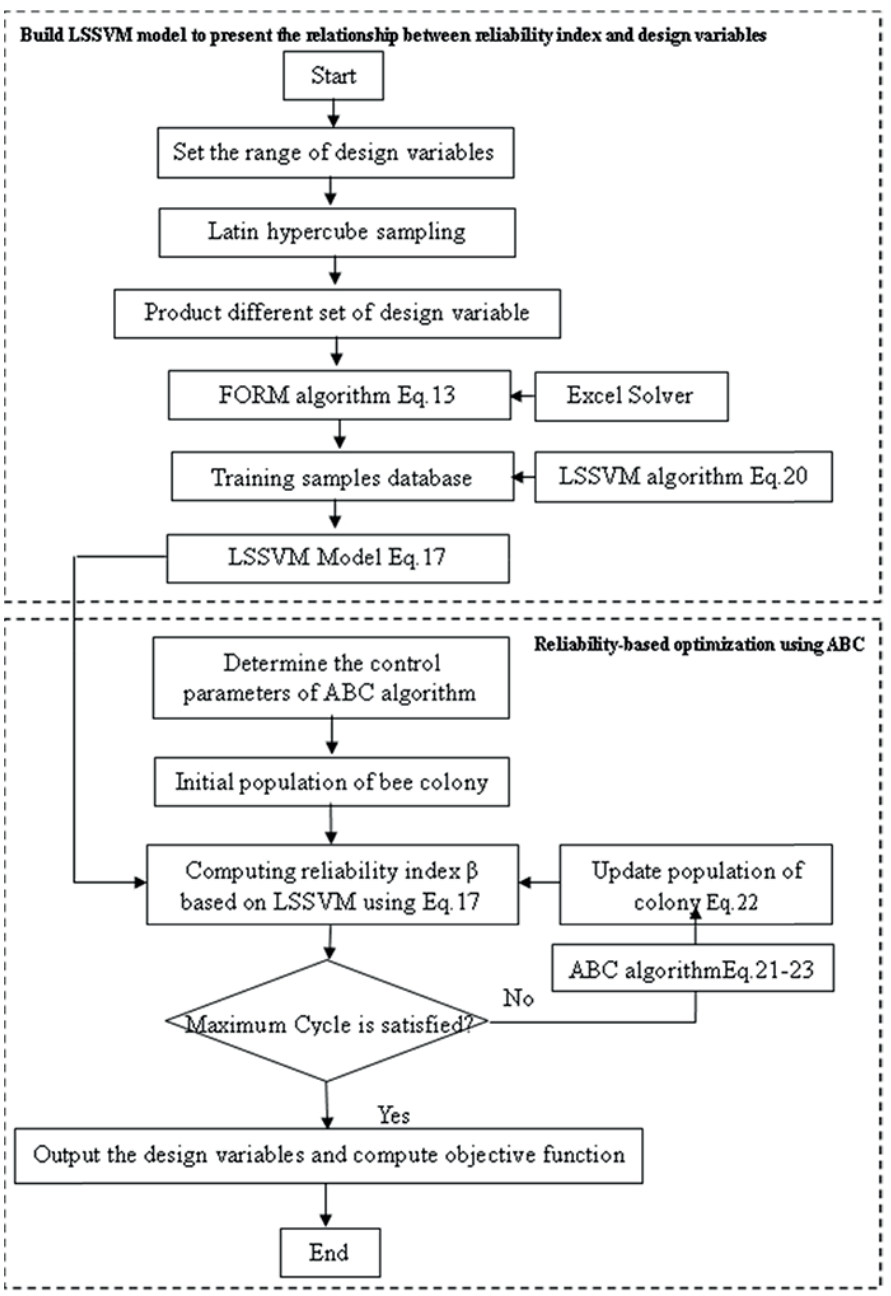

Fig. 4 Flowchart of LSSVM/ABC-based reliability optimization

\section{Illustrative examples}

The reliability and optimization of rockbolt parameters were evaluated by the proposed method for a $2 \mathrm{~m}$ radius circular tunnel reinforced by grouted rockbolts. The elastic modulus $(E)$ of the rock mass, in-situ stress $\left(\sigma_{0}\right)$ and internal pressure $p_{f}$ were the random variables (see Table 1). The internal pressure $p_{f}$ represents the support provided by the tunnel face at the time the rockbolts are installed. Poisson's ratio is 0.3 . The design variables are the rockbolt length $\left(L_{b}\right)$, distance of rockbolts out of plane $(D)$ and the number of rockbolts in the tunnel section $\left(n_{b}\right)$.The diameter of all rockbolts is $25 \mathrm{~mm}$; elastic modulus is $210 \mathrm{GPa}$. The displacement criterion of the tunnel wall and the failure criterion of the rockbolts were investigated. In this study, the value of $\varepsilon_{L}$ and $\sigma_{r}^{T}$ were taken to be 0.005 and 500 respectively. The objective function is the total length of all rockbolts. The reliability index constraints of the deformation and strength performance function are 3.0 and 2.0 respectively.

Table 1 Random variables and statistical properties

\begin{tabular}{lccc}
\hline Variables & Distribution & Mean & Standard Deviation \\
\hline $\mathrm{E}(\mathrm{MPa})$ & Normal & 270.73 & 27 \\
$\sigma_{0}(\mathrm{MPa})$ & Normal & 1 & 0.2 \\
$p_{f}$ & Normal & 0.7 & 0.07 \\
\hline
\end{tabular}

To determine the support capability of the rockbolts, the ABC parameters SN/2 and MCD were chosen as 30 and 50 respectively. The parameter $\gamma$ of LSSVM was 0.001 . The RBF kernel was adopted, with $\sigma=20 ; 300$ training samples and 100 testing samples were built using LHS. Comparisons between reliability index results obtained by FORM and results predicted by LSSVM are shown in Fig. 5. The comparisons indicate that LSSVM effectively represented the nonlinear relationship between the design variables and the reliability index for both performance functions, i.e. tunnel wall deformation and rockbolt strength. The optimal parameters for the rockbolts are listed in Table 2. The values of the design variables obtained by ABC agreed well with those from FORM. The reliability index meets the constraint requirements. The objective function is slightly higher than that by FORM (relative error $<0.7 \%$ ). In addition, the running time of the proposed method was about $170.45 \mathrm{~s}$, which was far less than the $1305.23 \mathrm{~s}$ taken by the FORM method. This demonstrates that, for this example, the proposed method dramatically improved the efficiency of RBO while maintaining high accuracy. Variations in the fitness and design variables are shown in Figs. 6 and 7. Fig. 8 shows the variation of population in different cycles. 
Table 2 Optimization results for rockbolt support

\begin{tabular}{|c|c|c|c|c|c|c|c|}
\hline \multirow{2}{*}{ Method } & \multicolumn{3}{|c|}{ Design variables } & \multicolumn{2}{|c|}{ Reliability index } & \multirow[t]{2}{*}{ Objective function (m) } & \multirow[t]{2}{*}{ Time (s) } \\
\hline & $L_{b}(\mathrm{~m})$ & $n_{b}$ & D (m) & G1 & G2 & & \\
\hline FORM by ABC & 1.0 & 26.176 & 0.629 & 3.368 & 2.001 & 41.640 & 1305.230 \\
\hline LSSVM model by Solver & 1.0 & 28.113 & 0.673 & $3.407 \mathrm{a} / 3.370 \mathrm{~b}$ & $2.000 \mathrm{a} / 2.008 \mathrm{~b}$ & 41.746 & - \\
\hline LSSVM model by ABC & 1.0 & 27.329 & 0.652 & $3.273 \mathrm{a} / 3.373 \mathrm{~b}$ & $2.054 \mathrm{a} / 2.022 \mathrm{~b}$ & 41.930 & 170.450 \\
\hline
\end{tabular}

Notes: a. Computed using LSSVM model; b. Computed using FORM.
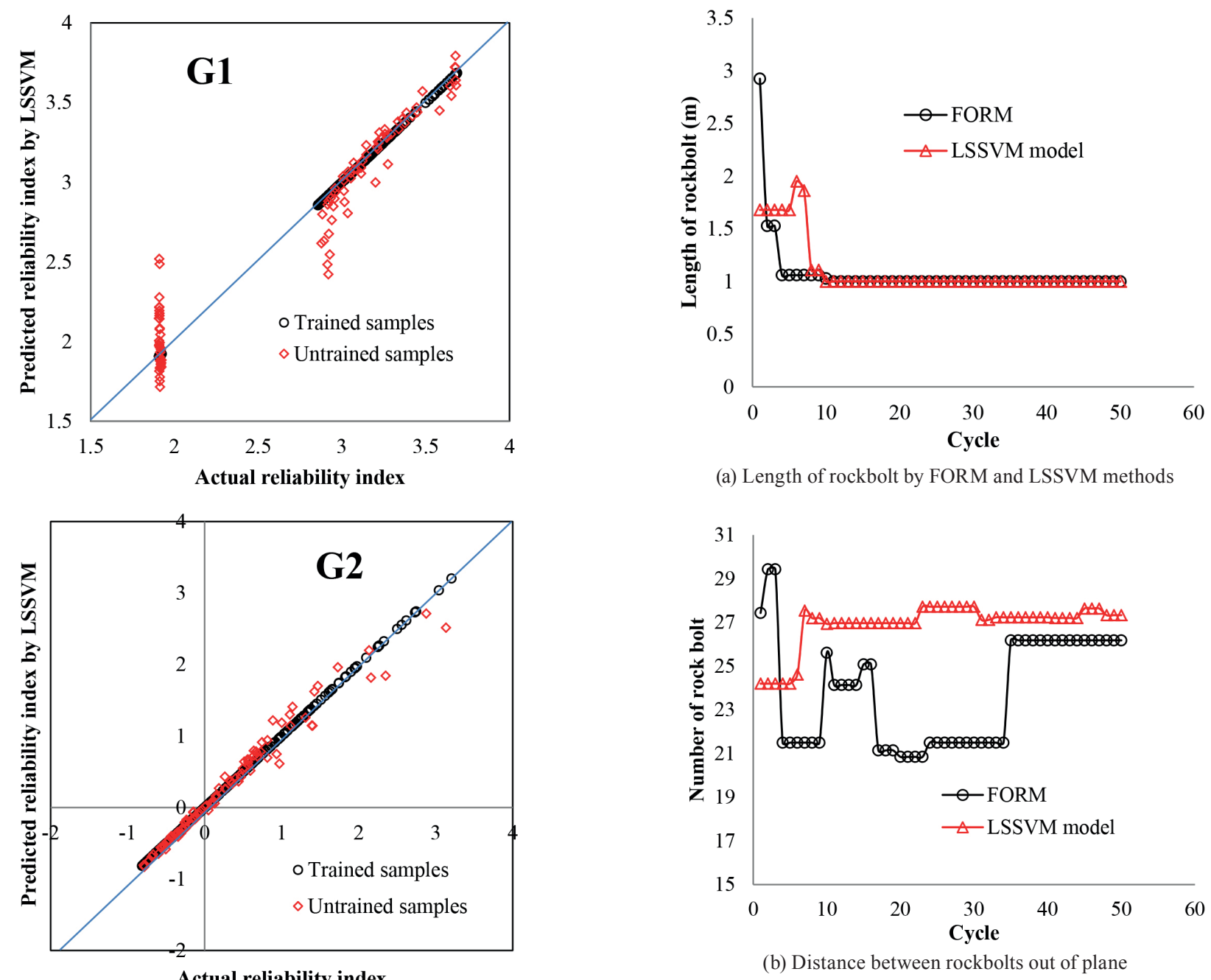

(a) Length of rockbolt by FORM and LSSVM methods

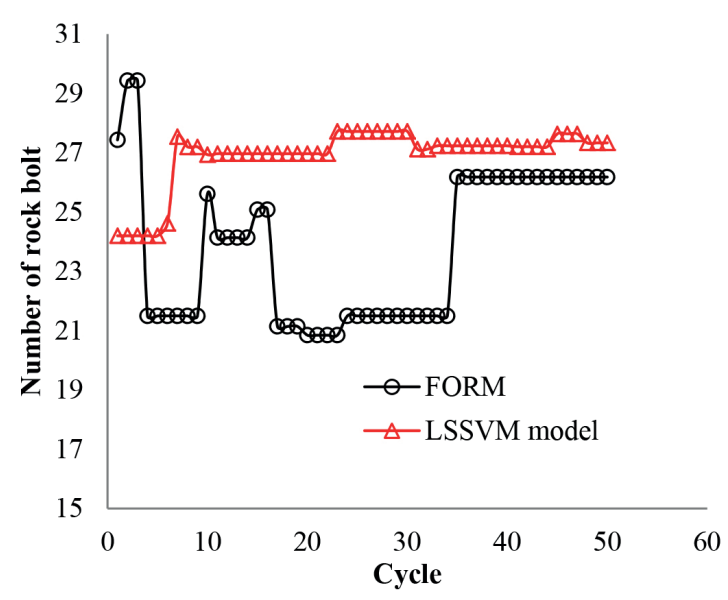

(b) Distance between rockbolts out of plane

Fig. 5 Comparison of reliability index by FORM and LSSVM methods

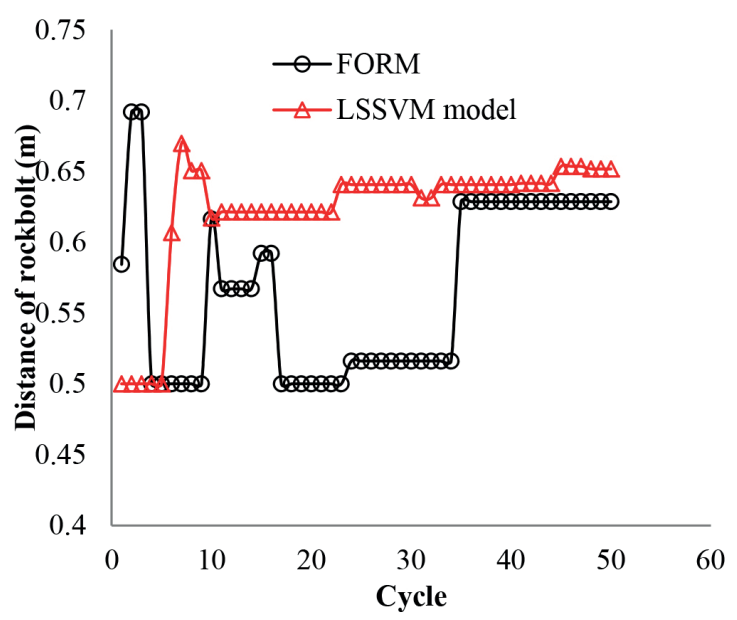

(c) Number of rockbolts in plane

Fig. 7 Variation of design variables: (a) length of rockbolt; (b) distance between rockbolts out of plane; (c) number of rockbolts in plane 

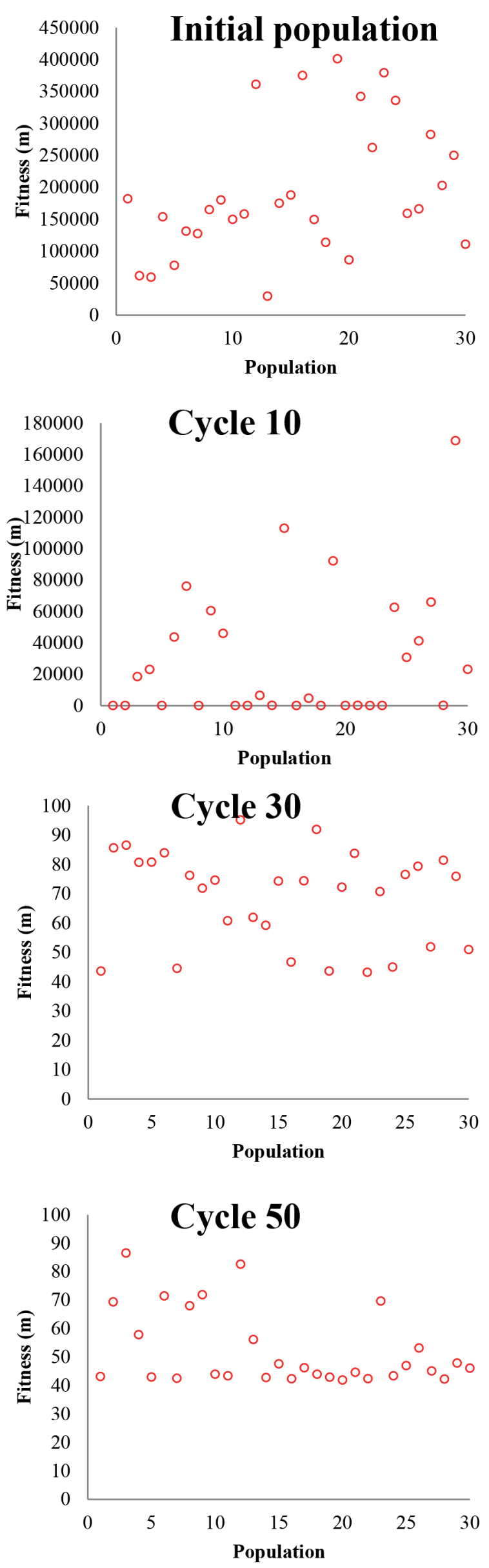

Fig. 8 Fitness variation of population in different cycles

\section{Summary and conclusions}

In this paper, a LSSVM/ABC-based RBO method that takes uncertainty into account is proposed for the design of ageotechnical system, in this case a circular tunnel in rock with rockbolt reinforcement. Results show that the proposed method can obtain accurate solutions with dramatically improved computing efficiency. The results were verified against an analytical solution. In fact, the proposed method can also be used fora tunnel with a numerical solution. The ABC method was used in the demonstration case, but any search optimization method could be used, such as a genetic algorithm, gradient based methods, etc.

\section{Acknowledgements}

Supports by the Program for Innovative Research Team (in Science and Technology) in University of Henan Province (No.15IRTSTHN029) and Doctorate Fund of Henan Polytechnic University (No.B2009-96) are gratefully acknowledged. The authors also appreciate the anonymous referees for their valuable suggestions and questions.

\section{References}

[1] Barton, N., Lien, R., Lunde, J. "Engineering classifications of rock masses for the design of tunnel support”. Rock Mechanics, 6(4), pp.189-236. 1974. https://doi.org/10.1007/BF01239496

[2] Bieniawski, Z.T. "Engineering classification of rock masses". Civil Engineer in South Africa, 15(12), pp. 335-344. 1973.

[3] Goodman, R. E., Shi, G. "Block Theory and its Application in Rock Engineering”. Prentice Hall. 1985.

[4] Wang, Y. "Reliability-based economic design optimization of spread foundations". Journal of Geotechnical and Geoenvironmental. Engineering, 135(7), pp. 954-959. 2009. https://doi.org/10.1061/(ASCE) GT.1943-5606.0000013

[5] Wang,Y. "Reliability-based design of spread foundations by Monte Carlo simulations". Géotechnique, 61(8), pp. 677-85. 2011. https://doi. org/10.1680/geot.10.P.016

[6] Zhang, J., Zhang, L.M. and Tang, W.H. "Reliability-based optimization of geotechnical systems". Journal of Geotechnical and Geoenvironmental Engineering, 137(12), pp.1211-1221. 2011. 10.1061/(ASCE)GT.19435606.0000551.

[7] Yang, I. T., Hsieh, Y. H. "Reliability-based design optimization with discrete design variables and non-smooth performance functions: AB-PSO algorithm". Automation in Construction, 20(5), pp.610-619. 2011. https:// doi.org/10.1016/j.autcon.2010.12.003

[8] Samer. A. B., Abdallah, I. H.M., Raed, H. T. "Reliability-based optimization of laterally loaded piles”. Structral Safety, 21(1), pp.45-64. 1999. https://doi.org/10.1016/S0167-4730(99)00004-1

[9] Irfan, K., Kurt, M. "Reliability-based design optimization for elastoplastic mechanical structures". Computers and Structures, 85(10), pp. 615-625. 2007. https://doi.org/10.1016/j.compstruc.2006.08.076

[10] Younes, Aoues.,and Alaa, Chateauneuf. "Benchmark study of numerical methods for reliability-based design optimization". Structural and Multidisciplinary Optimization, 41(2), pp. 277-294. 2010. https://doi. org/10.1007/s00158-009-0412-2

[11] Marcos, A.V., Gerhart, I. S. "A survey of approaches for reliability-based optimization". Structural and Multidisciplinary Optimization, 42(5), pp. 645-663. 2010. https://doi.org/10.1007/s00158-010-0518-6 
[12] Song, C. Y., Lee, J. "Reliability-based design optimization of knuckle component using conservative method of moving least squares meta-models". Probabilistic Engineering.Mechanics, 26(2), pp. 364-379. 2011. https://doi.org/10.1016/j.probengmech.2010.09.004

[13] Song, C. Y., Lee, J., Choung, J. M. "Reliability-based design optimization of an FPSO riser support using moving least squares response surface meta-models". Ocean Engineering, 38(2-3), pp. 304-318. 2011. https://doi.org/10.1016/j.oceaneng.2010.11.001

[14] Gomes, H. M., Armando, M. A., Paulo, A. M. L. "Reliability based optimization of laminated composite structures using genentic algorithms and artifical neural networks". Structural Safety, 33(3), pp. 186-195. 2011. https://doi.org/10.1016/j.strusafe.2011.03.001

[15] Gen, M., Yun, Y. "Soft computing approach for reliability optimization: State-of-the-art survey". Reliability Engineering and System Safety, 91(9), pp. 1008-1026. 2006. https://doi.org/10.1016/j.ress.2005.11.053

[16] Lee, I., Shin Jaekwan, K. K., Choi, K. K. "Equivalent target probability of failure to convert high-reliability model to low-reliability model for efficiency of sampling-based RBDO". Structural and Multidisciplinary Optimization, 48(2), pp. 235-248. 2006. https://doi.org/10.1007/s00158013-0905-x

[17] Karaboga, D. "An idea based on honey bee swarm for numerical optimization”. Technical Report-TR06, Erciyes University, Engineering Faculty, Computer Engineering Department. 2005.
[18] Carranza-Torres, C. "Analytical and numerical study of the mechanics of rockbolt reinforcement around tunnels in rock masses". Rock Mechanics and Rock Engineering, 42(2), pp.175-228. 2009. https://doi.org/10.1007/ s00603-009-0178-2

[19] Hasofer, A.M., Lind, N. C. "Exact and invariant second moment code format”. Journal of Engineering Mechanics, 100(1), pp. 111-121. 1974.

[20] Low, B. K., Tang, W. H. "Efficient spreadsheet algorithm for first-order reliability method”. Journal of Engineering Mechanics, 133(12), pp. 13781387. 2007. https://doi.org/10.1061/(ASCE)0733-9399(2007)133:12(1378)

[21] Suykens, J. A. K., Vandewalle, J. "Least squares support vector machine classifiers”. Neural Processing Letters, 9(3), pp. 293-300. 1999. https:// doi.org/10.1023/A:1018628609742

[22] Karaboga, D., Basturk, B. "On the performance of artificial bee colony (ABC) algorithm”. Applied Soft Computing, 8(1), pp. 687-697. 2008. https://doi.org/10.1016/j.asoc.2007.05.007

[23] Karaboga, D. and Ozturk, C. "A novel clustering approach: Artificial Bee Colony (ABC) algorithm". Applied Soft Computing, 11(1), pp. 652 657. 2011. https://doi.org/10.1016/j.asoc.2009.12.025

[24] Mckay, M.D., Conover, W. J., Beckman, R. J. "A comparison of three methods for selecting values of input variables in the analysis of output from a computer code". Technometrics, 21(2), pp. 239-245. 1979. https:// doi.org/10.2307/1268522 